\title{
Verbos transitivos que presentan una variante intransitiva no pronominal con interpretación de propiedad y su tratamiento lexicográfico*
}

\author{
Elena Felíu Arquiola y Marta Torres Martínez \\ Universidad de Jaén
}

\section{INTRODUCCIÓN}

En este artículo nos proponemos un triple objetivo. En primer lugar, realizaremos un estudio descriptivo de los verbos transitivos que pueden presentar una variante intransitiva no pronominal con interpretación de propiedad en español (apartado 2). Nos referimos a verbos como lavar o planchar que, además de poseer una variante transitiva eventiva (1a, 1b), presentan también la posibilidad de aparecer en construcciones intransitivas no pronominales con interpretación no eventiva en las que el argumento Tema o Paciente realiza la función sintáctica de sujeto y el argumento Agente queda inexpresado (2a, 2b):

(1) Variante transitiva eventiva:

a. Luis ha lavado la funda del sofá

b. Julia planchó tres camisas de algodón

* Una primera versión de este trabajo se presentó en el XXXIII Simposio de la Sociedad Española de Lingüística (Universitat de Girona, 16-19 de diciembre de 2003). La parte de la investigación realizada por Marta Torres Martínez se ha llevado a cabo gracias a una beca predoctoral de la Junta de Andalucía (convoc. 2003) mientras que la investigación realizada por Elena Felíu Arquiola se ha visto apoyada por el proyecto de investigación I+D "Límites intercategoriales: las categorías híbridas. Teoría, descripción y aplicaciones", Ministerio de Educación y Ciencia, Plan Nacional de Investigación Científica, Desarrollo e Innovación Tecnológica 2004-2007 (ref.: HUM2004-04235C02-01/FILO). 
(2) Variante intransitiva no pronominal no eventiva:

a. La funda de este sofá lava bien

'La funda de este sofá es lavable / La funda de este sofá se puede lavar'

b. Las camisas de algodón planchan bien

'Las camisas de algodón son planchables / Las camisas de algodón se pueden planchar'

En segundo lugar, en el apartado 3 analizaremos el tratamiento lexicográfico que estos verbos reciben en los principales diccionarios generales de español (DRAE-2001, DEA, DUE, SALAMANCA ${ }^{1}$ ). En concreto, nos interesa estudiar si esta variante intransitiva con interpretación de propiedad que presentan verbos como lavar, planchar, abrir o cerrar aparece codificada en los diccionarios de español como una acepción independiente de la acepción eventiva o si, por el contrario, esta variante intransitiva no recibe atención en los repertorios lexicográficos generales. De igual modo, nos interesa estudiar si en un mismo diccionario se codifica la variante intransitiva de propiedad en el caso de un verbo pero no en el caso de otros verbos que también la permiten y si esta variante intransitiva de propiedad recibe siempre un mismo tipo de definición, esto es, nos interesa estudiar el grado de coherencia que presentan los principales diccionarios generales de español en relación con el tratamiento lexicográfico de estos verbos.

En tercer lugar, en el apartado 4 presentaremos una propuesta de codificación lexicográfica de la variante intransitiva con interpretación de propiedad que permiten ciertos verbos habitualmente transitivos eventivos que subsane las deficiencias encontradas al realizar la revisión del tratamiento lexicográfico de estos verbos. Las principales conclusiones alcanzadas en este artículo se presentan en el apartado 5. Finalmente, en un apéndice se consignan ejemplos documentados en español actual

1 A pesar de que el Diccionario Salamanca no es propiamente un diccionario general, sino un repertorio lexicográfico dirigido a la enseñanza del español, hemos decidido incluirlo entre las obras revisadas, ya que, como veremos en el apartado 3.3, presenta algunas particularidades interesantes en relación con el tratamiento de los verbos objeto de estudio. 
de la variante intransitiva no pronominal con interpretación de propiedad que permiten algunos verbos de nuestra lengua.

Este trabajo resulta novedoso por dos motivos. Por una parte, la construcción objeto de estudio, que presenta la variante no pronominal estativa de verbos generalmente transitivos eventivos (Esta camisa lava bien), no ha recibido mucha atención en la bibliografía especializada en español, frente al mayor interés que han despertado las construcciones relacionadas denominadas "mediopasivas" o "medias" (Esta camisa se lava fácilmente). Por otra parte, no conocemos ningún estudio en el que se aborde el tratamiento lexicográfico de estos verbos. La idea de revisar el tratamiento que una clase determinada de verbos recibe en los diccionarios generales de español no es nueva, sin embargo. Así, por ejemplo, Azorín Fernández y Martínez Linares (2000 y 2001) han estudiado la evolución del tratamiento lexicográfico de los verbos pronominales tanto en el DRAE como en diccionarios generales no académicos. Nuestro trabajo coincide con los dos artículos mencionados en el hecho de que nos interesa analizar el tratamiento lexicográfico de una clase determinada de verbos, pero se diferencia en dos aspectos fundamentales: por una parte, no realizaremos un recorrido diacrónico por las sucesivas ediciones de un diccionario, sino que llevaremos a cabo una comparación de tipo sincrónico entre varios diccionarios distintos; por otra, nos centraremos en un grupo de verbos mucho más restringido que la clase de los verbos pronominales, denominación bajo la que se incluyen, como es sabido, clases léxicas semánticamente diversas, que comparten la particularidad morfosintáctica de construirse -bien de manera opcional, bien de manera obligatoria- con un clítico con los mismos rasgos de persona y número que el sujeto.

\section{DESCRIPCIÓN Y ANÁLISIS}

\subsection{VERBOS QUE PERMITEN UNA VARIANTE INTRANSITIVA NO PRONOMINAL CON INTERPRETACIÓN DE PROPIEDAD}

En (3) presentamos varios ejemplos de la clase de verbos que hemos elegido como objeto de estudio: 
(3) a. Las camisas de algodón planchan bien

b. Esta puerta no cierra

c. Los garbanzos congelan bien

Como podemos observar, se trata de verbos habitualmente transitivos (planchar, cerrar, congelar) que permiten también una variante intransitiva no pronominal cuyo sujeto se corresponde con el sintagma nominal que funcionaría como objeto directo en la variante transitiva. Las oraciones de (3) reciben una interpretación de propiedad con significado de posibilidad: 'Las camisas de algodón se pueden planchar' (3a), 'Esta puerta no se puede cerrar' (3b), 'Los garbanzos se pueden congelar' (3c).

Como ya afirmamos en la introducción, este tipo de construcción estativa no pronominal formada a partir de verbos transitivos eventivos no ha recibido mucha atención en la bibliografía especializada sobre el español. Encontramos referencias aisladas a algunos verbos que permiten esta variante estativa no pronominal generalmente en trabajos que se centran en el estudio de fenómenos de mayor amplitud, como la transitividad en español o las construcciones aspectuales habituales o estativas.

En lo que respecta a los trabajos del primer tipo, esto es, aquellos en los que se aborda el estudio de la transitividad en español, podemos mencionar el libro de Cano Aguilar (1981:237), en el que se menciona el hecho de que los verbos abrir y cerrar pueden ser usados intransitivamente con un significado de propiedad próximo al de las construcciones mediopasivas en oraciones como Esta puerta no \{abre / cierra\} bien.

Por otra parte, entre los trabajos que tratan las construcciones aspectuales habituales o estativas en español, se encuentra un artículo de Rodríguez Espiñeira (1990) sobre predicados habituales, así como un reciente artículo de Fernández, Vázquez y Martí (2002) sobre alternancias diatéticas relacionadas con el aspecto.

Rodríguez Espiñeira (1990: 206-207), sitúa ejemplos como Estas ventanas cierran con dificultad, Estas habas no cuecen bien y Este tipo de botellas abren mal entre las expresiones relacionadas con la noción de habitualidad, bajo la denominación de "construcciones pronominales", sin tener en cuenta que en las tres oraciones mencionadas no apa- 
recen verbos en construcción pronominal. Por su parte, Fernández, Vázquez y Martí (2002: 396-397), señalan que, en ocasiones, la construcción mediopasiva o media, que también recibe una interpretación de propiedad, puede formarse bien con un verbo en construcción pronominal (Este tejido se seca bien), bien con un verbo intransitivo no pronominal (Este tejido seca bien). En la nota 11 las autoras afirman que "la ausencia del pronombre sólo es válida en la interpretación media y no en la eventiva correspondiente (*Este tejido secó rápido)", frente a la construcción pronominal, que puede recibir una interpretación tanto no eventiva (Este tejido se seca rápidamente) como eventiva (Este tejido se secó rápidamente).

Consideramos que los verbos que pueden presentar esta variante intransitiva no pronominal con interpretación de propiedad están léxicamente restringidos: en principio, parece tratarse de verbos transitivos cuyo argumento externo puede ser tanto un Agente (4a) como una Causa (4b), y cuyo argumento interno se ve siempre afectado por la acción verbal en el sentido de que experimenta un cambio de estado físico, tal como se observa en los ejemplos de $(4)^{2}$ :

(4) a. Verbos agentivos ${ }^{3}$ : cocinar, coser, exprimir, lavar, limpiar, picar, planchar, rallar.

b. Verbos causativos ${ }^{4}$ : abrir, ajustar, arrugar, asar, doblar, cerrar, cocer, congelar, descongelar, dorar, freír, fruncir, rizar, secar, teñir, tostar.

Sin embargo, la caracterización que acabamos de presentar no es suficientemente restrictiva, ya que existen numerosos verbos transitivos

2 La lista de (4) no pretende ser exhaustiva. Es probable que otros verbos transitivos del español permitan igualmente una variante intransitiva no pronominal con interpretación de propiedad. De hecho, la aceptación de este tipo de construcciones puede variar de un hablante a otro.

3 Se trata, por tanto, de verbos que no participan en la alternancia causativa/incoativa $(*$ La camisa se lavó (sola)).

4 Se trata, por tanto, de verbos que participan en la alternancia causativa/incoativa ( $L a$ puerta se abrió (sola)). 
cuyo argumento externo es un Agente o una Causa y cuyo argumento interno experimenta un cambio de estado físico que no poseen una variante intransitiva no pronominal con interpretación de propiedad. Así, por ejemplo, escribir y pintar responden a la caracterización anterior -son verbos agentivos cuyo argumento interno experimenta un cambio de estado físico- $y$, sin embargo, carecen de dicha variante intransitiva, como se observa en los ejemplos de (5):

(5) a. *Este libro escribe bien 'Este libro se puede escribir'

b. *Este lienzo pinta bien 'Este lienzo se puede pintar'

Entre los verbos transitivos escribir y pintar existe una diferencia importante: mientras que escribir es un verbo de creación, esto es, la entidad denotada por su argumento interno cobra existencia como resultado de la acción verbal, pintar en una oración como María pintó el lienzo puede ser interpretado no como verbo de creación, sino como un verbo de objeto afectado físicamente. En esta interpretación pintar se aproxima a verbos como lavar o planchar. Sin embargo, a diferencia de estos dos verbos, pintar no posee una variante intransitiva no pronominal con interpretación de propiedad. Otros verbos que, como pintar, implican la afectación física del objeto pero carecen de la variante intransitiva que aquí nos interesa son romper, manchar y arreglar, como se observa en (6), aunque se diferencian de pintar en que son verbos causativos más que puramente agentivos:

(6) a. romper: *Este material rompe bien, *Este material no rompe.

b. manchar: *Este tejido mancha bien; *Este tejido no mancha.

c. arreglar: *Los aparatos eléctricos arreglan bien; *Este ventilador no arregla.

En nuestra opinión, no es posible delimitar una clase léxica concreta que englobe a todos los verbos que permiten esta construcción estativa no pronominal. Junto con verbos causativos como abrir o cerrar encontramos también verbos agentivos como coser, lavar o planchar, que no pertenecen a la misma clase léxica. Por otra parte, un verbo causativo como romper, con un comportamiento léxico-sintáctico muy similar al de abrir o cerrar -participa en la alternancia causativo-incoativa (La pedrada rompió el cristal / El cristal se rompió)-, no presenta esta variante 
intransitiva no pronominal con interpretación de propiedad, tal como se mostró en (6a). Por otra parte, parece que en ocasiones esta variante intransitiva no pronominal con interpretación de propiedad se documenta con cierta frecuencia en el léxico especializado de ciertas actividades y profesiones, como sucede por ejemplo con el léxico relacionado con actividades domésticas. Así, entre los verbos para los que hemos documentado una variante intransitiva no pronominal con interpretación de propiedad se encuentran cocinar, congelar, descongelar, lavar, planchar, secar, teñir, etc. Podríamos pensar que nos encontramos ante un rasgo lingüístico asociado a factores sociales como la profesión o el género, habitualmente no identificado como tal en los estudios sociolingüísticos sobre el español. Dejamos pendiente para futuras investigaciones el estudio desde una perspectiva sociolingüística del empleo de verbos como lavar, coser o planchar en su variante intransitiva no pronominal con interpretación de propiedad, cuestión de evidente interés que las limitaciones de espacio nos impiden abordar en este artículo.

Aunque los verbos elegidos como objeto de estudio no han sido tratados con detalle en los estudios lingüísticos del español de forma conjunta, la construcción mediopasiva -si seguimos la tradición española- o construcción media ${ }^{5}$-en la tradición anglosajona-, ejemplificada en (7), sí ha sido el objeto de interés de los estudios gramaticales desde finales de los años 70 . Entre los trabajos más recientes podemos citar los artículos de García Negroni (1996 [2002]), Mendikoetxea (1999, 2000), Catalá, Martí y Vallhonrat (2002), Fernández, Vázquez y Martí (2002) y Sánchez López (2002).

5 Como es sabido, las oraciones medias han sido ampliamente estudiadas en el caso de otras lenguas como el inglés. En dicha lengua, las construcciones medias se forman precisamente mediante la variante intransitiva estativa de un verbo transitivo eventivo. Sin embargo, se trata de un proceso que afecta a un número de verbos mucho mayor que la alternancia que aquí estudiamos, que se encuentra restringida a unas cuantas piezas léxicas verbales. El equivalente español de las oraciones medias del inglés está formado no tanto por verbos intransitivos no pronominales como por verbos acompañados por el clítico se (Esta camisa se lava fácilmente), opción más extendida y menos marcada que la variante no pronominal (Esta camisa lava bien). Para un estudio detallado de las oraciones medias del inglés, remitimos al trabajo de Levin (1993). 
(7) Construcción mediopasiva o media:

a. Este tejido se seca fácilmente

b. Los puentes de madera se construyen fácilmente

En este tipo de construcción, el sintagma nominal que desempeñaría la función sintáctica de objeto directo en la variante transitiva del verbo, funciona como sujeto en la variante intransitiva, mientras que el sintagma que realizaría la función sintáctica de sujeto en la variante transitiva, no aparece en la variante intransitiva.

Los verbos intransitivos no pronominales que nos ocupan comparten con las construcciones mediopasivas no sólo la interpretación de propiedad, sino también la tendencia a presentar algún tipo de modificación adverbial, bien adverbios de modo (8a), bien la negación (8b), aunque es posible encontrar casos en los que la modificación adverbial no resulta necesaria $(8 \mathrm{c})$ :

(8) a. Este tejido lava bien

b. Este tejido no lava

c. Este tejido arruga (mucho)

Sin embargo, no todos los adverbios que encontramos en las construcciones mediopasivas pueden también aparecer con los verbos intransitivos no pronominales con interpretación de propiedad. Así, por ejemplo, mientras bien y mal dan lugar a construcciones gramaticales cuando se combinan con los verbos intransitivos no pronominales con interpretación de propiedad, el adverbio fácilmente -empleado con frecuencia en los estudios sobre construcciones mediopasivas- da lugar a construcciones menos aceptables 6 , como se observa en los ejemplos de (9), frente a los casos de construcciones mediopasivas de (10):

6 No podemos dejar de señalar que en el $D E A$ figura como cita ilustrativa del uso intransitivo no pronominal con interpretación de propiedad del verbo lavar una oración en la que aparece el adverbio fácilmente: Los jerseys Crilenka lavan muy fácilmente (Ya 9.12.72, $D E A$, s.v.), tal como se observa en el artículo lexicográfico correspondiente, que presentamos más adelante (véase (20e)). En nuestra opinión, la combinación de fácilmente con esta variante intransitiva de propiedad del verbo lavar, si no agramatical, sí resulta cuanto menos extraña, pese a que se documente algún ejemplo de uso. 
(9) a. Las camisas de algodón \{lavan / planchan \} bien

b. ??Las camisas de algodón \{lavan / planchan \} fácilmente

(10) a. Las camisas de algodón se \{lavan / planchan \} fácilmente

b. Los puentes de madera se construyen fácilmente

Consideramos que este hecho puede estar relacionado con la ausencia de un argumento implícito en el caso de los verbos intransitivos no pronominales con interpretación de propiedad. Se ha propuesto que en las construcciones mediopasivas del español existe un argumento Agente sobreentendido, como sucede en el trabajo de Mendikoetxea (1999: 1655) ${ }^{7}$. En nuestra opinión, la restricción que afecta al tipo de adverbio que modifica a los verbos intransitivos no pronominales con interpretación de propiedad puede explicarse si se considera que en el caso de tales verbos no existe ningún argumento agentivo implícito o sobreentendido, sino que se trata de verbos monoargumentales tanto sintáctica como semánticamente. En cambio, en la construcción mediopasiva o media existiría un Agente sobreentendido que legitimaría la aparición de adverbios como fácilmente o difícilmente, que indican la manera en que dicho Agente lleva a cabo la acción. La misma restricción que presentan los verbos intransitivos no pronominales con interpretación de propiedad en relación con el tipo de adverbios permitidos parece operar, si bien de forma más estricta, sobre otros predicados de propiedad que claramente carecen de todo tipo de interpretación agentiva, como los presentados en (11), que resultan agramaticales combinados con adverbios como fácilmente:

(11) a. María sabe inglés \{bien / *ácilmente $\}$

b. María conoce $\{$ bien / *fácilmente $\}$ su ciudad

Para finalizar esta descripción de las construcciones en las que aparecen los verbos intransitivos no pronominales con interpretación de propiedad, hay que señalar que estos verbos sólo son compatibles con

7 No todos los autores aceptan la existencia de un Agente sobreentendido en las construcciones mediopasivas del español. Así, por ejemplo, Sánchez López (2002: 69) niega que exista un argumento Agente implícito en las construcciones mediopasivas. 
tiempos imperfectivos (12a, 12b), mientras que no suelen aparecer en tiempos perfectivos $(12 \mathrm{c}, 12 \mathrm{~d})$, tal como ya ha sido señalado por Fernández, Vázquez y Martí (2001: 397, n. 11):

(12) a. Las camisas de algodón lavan bien

b. Los tejidos antiguos lavaban bien

c. ?Las camisas de algodón han lavado bien

d. ?Ayer este tejido lavó bien

Este hecho se relaciona con la interpretación de propiedad de estos verbos, que les impide aparecer en tiempos de aspecto perfectivo a no ser que estén acompañados por adverbios aspectuales del tipo de siempre o nunca, como se puede observar en (13):

(13) a. Este tejido siempre ha lavado bien

b. Esta camisa nunca ha arrugado

De nuevo, otros predicados que expresan propiedades como saber inglés bien o conocer bien su ciudad presentan el mismo comportamiento en este sentido, como queda reflejado en los ejemplos de (14):

(14) a. María *(siempre) ha sabido inglés bien

b. María *(siempre) ha conocido bien su ciudad

En resumen, consideramos que sólo algunos verbos poseen variantes intransitivas no pronominales con interpretación de propiedad. Se trata siempre de verbos transitivos con un argumento externo Agente o Causa y un argumento interno afectado físicamente. Sin embargo, la caracterización anterior enuncia una condición necesaria pero no suficiente: existen numerosos verbos transitivos con un argumento externo Agente o Causa y un argumento interno afectado físicamente que no permiten esta variante intransitiva no pronominal de propiedad, como romper, manchar o arreglar ${ }^{8}$. Consideramos, pues, que no resulta posible delimitar una clase léxica concreta de verbos sometidos a esta alternancia. Parece más bien que esta doble posibilidad -variante transitiva eventiva / variante intransitiva no pronominal estativa- afecta a ciertos verbos

Véanse en este sentido los ejemplos de (6). 
concretos pertenecientes a distintas clases léxicas. Por otra parte, los adverbios que aparecen normalmente con estos verbos intransitivos son semánticamente más restringidos que los que aparecen en las construcciones mediopasivas, restricción que parece explicarse si se asume que los verbos intransitivos no pronominales con interpretación de propiedad no implican la existencia de un Agente sobreentendido, a diferencia de lo que se propone para las construcciones mediopasivas. Sin embargo, al igual que estas construcciones, los verbos intransitivos no pronominales con interpretación de propiedad sólo son compatibles con tiempos verbales de aspecto imperfectivo, salvo que estén acompañados por un adverbio aspectual del tipo de siempre.

\subsection{UN CASO DE POLISEMIA LÉXICA}

A la luz del estudio descriptivo presentado en el subapartado anterior, consideramos que esta alternancia entre la variante transitiva eventiva y la variante intransitiva no pronominal con interpretación de propiedad puede ser analizada como un caso de polisemia léxica, es decir, como la existencia de dos variantes léxicas de una serie de verbos (lavar, secar, planchar, etc.) una de ellas transitiva eventiva y la otra intransitiva no pronominal con interpretación de propiedad.

Un posible análisis alternativo consistiría en proponer la existencia de una regla léxica de intransitivización de un verbo transitivo que llevara aparejado un cambio semántico de evento a propiedad. Sin embargo, esta alternancia está muy restringida desde el punto de vista léxico. Se limita, como hemos mostrado en el apartado 2.1, a ciertos verbos transitivos cuyo argumento externo es un Agente o una Causa y cuyo argumento interno se ve afectado físicamente por la acción verbal. Por este motivo, consideramos que no nos encontramos ante el resultado de la aplicación de una regla léxicosemántica de manera productiva, sino ante casos concretos de polisemia léxica.

Un caso bien distinto es, en nuestra opinión, la construcción mediopasiva o media. Algunos autores, como por ejemplo Mendikoetxea (1999: 1656), consideran que en español únicamente dan lugar a construcciones mediopasivas los verbos transitivos cuyo argumento externo 
es un Agente (Los puentes de madera se construyen con facilidad), mientras que quedan descartados los verbos transitivos cuyo argumento externo es un Experimentante ( ${ }^{*}$ Las acelgas se detestan en el momento de probarlas por primera vez, apud Mendikoetxea 1999: 1656). Sin embargo, otros autores, como Catalá, Martí y Vallhonrat (2002: 376) y Felíu Arquiola (2004), consideran que los verbos cuyo argumento externo es un Experimentante también pueden dar lugar construcciones mediopasivas (Los planetas se ven fácilmente ${ }^{9}$, apud Catalá, Martí y Vallhonrat 2002: 376; Los ruidos de los vecinos se oyen fácilmente, apud Felíu Arquiola 2004). Desde nuestro punto de vista, la agramaticalidad de una oración como * Las acelgas se detestan en el momento de probarlas por primera vez (apud Mendikoetxea, 1999: 1656) no se debería al hecho de que el argumento externo de detestar sea un Experimentante (sobre esta misma cuestión, véase la nota 7), sino al hecho de que detestar es un predicado de estado que no puede dar lugar a una construcción como la mediopasiva, en la que un predicado eventivo se convierte en un predicado de propiedad, como se afirma en el trabajo de Felíu Arquiola (2004).

Si seguimos esta última hipótesis, la construcción mediopasiva del español impondría pocas restricciones a los verbos que pueden apare-

9 Mendikoetxea (1999: 1662) admite que un verbo como ver, cuyo argumento externo es un Experimentante, puede dar lugar a construcciones mediopasivas cuando el sujeto del que se predica tiene propiedades inherentes que permiten la interpretación de propiedad. En opinión de esta autora, una oración como Las luces reflectantes se ven fácilmente recibiría una interpretación mediopasiva (de propiedad), mientras que una oración como Las montañas se ven fácilmente recibiría una interpretación pasiva (eventiva). La diferencia residiría en el tipo de referente denotado por cada sujeto. Sin embargo, desde nuestro punto de vista (Felíu Arquiola 2004), ambas oraciones podrían ser consideradas mediopasivas. El referente del sintagma las montañas podría también ser entendido de tal manera que se le supusieran determinadas características que le permiten ser visto fácilmente. Por ejemplo, podríamos completar la segunda oración de la siguiente forma: Las montañas se ven fácilmente, pero las colinas no. Así pues, no vemos ninguna diferencia relevante entre este ejemplo y la oración Las luces reflectantes se ven fácilmente que, en opinión de Mendikoetxea (1999: 1662), sí puede recibir una interpretación mediopasiva (de propiedad). Para nosotros se trata, en ambos casos, de construcciones mediopasivas, esto es, con interpretación de propiedad. 
cer en ella: deben tratarse de verbos eventivos biargumentales, cuyo argumento externo puede ser tanto un Agente como un Experimentante. En este caso, podría resultar adecuado proponer una regla morfosemántica de formación de construcciones mediopasivas en español, tal como se ha propuesto para lenguas como el inglés o el holandés (Fagan 1988, 1992, Ackema y Schoorlemmer 1994, 1995), ya que se trata de un proceso muy regular que está gobernado por escasas restricciones léxicosemánticas. Remitimos al lector interesado a los trabajos citados tanto sobre otras lenguas (Fagan 1988, 1992, Ackema y Schoorlemmer 1994, 1995, por mencionar únicamente algunos de los trabajos en los que se adopta una aproximación léxica a las construcciones medias del inglés y del holandés) como sobre el español (García Negroni 1996 [2002], Mendikoetxea 1999, 2000, Catalá, Martí y Vallhonrat 2002, Fernández, Vázquez y Martí 2002, Sánchez López 2002, Felíu Arquiola 2004).

En lo que respecta a la variante intransitiva no pronominal con interpretación de propiedad de determinados verbos del español, objeto de estudio de este trabajo, nos parece más adecuado considerar que se trata de casos concretos de polisemia léxica, ya que, por una parte, la variante intransitiva no pronominal con interpretación de propiedad se encuentra muy restringida léxicamente -se da con un número muy reducido de verbos en comparación con la construcción mediopasiva- $\mathrm{y}$, por otra, su empleo no parece ser homogéneo entre los hablantes de español, cuestión que nos hemos limitado a apuntar en el apartado 2.1 y que requeriría de un estudio sociolingüístico y quizá también dialectal.

Para dar cuenta de las diferencias semánticas y morfosintácticas de la variante transitiva eventiva y la variante intransitiva no pronominal con interpretación de propiedad permitidas por verbos como lavar o planchar, proponemos las estructuras léxico-conceptuales recogidas en (15). En (15a) presentamos la estructura léxico-conceptual correspondiente a la variante transitiva eventiva, que puede ser agentiva o causativa ${ }^{10}$; por su parte, en (15b) presentamos la estructura léxico-conceptual correspondiente a la variante intransitiva con interpretación de propiedad:

10 Mediante las llaves ( \{\}$)$ tratamos de sintetizar la estructura léxico-semántica de los verbos agentivos y la de los verbos causativos en la misma representación. 
(15) a. Verbo transitivo eventivo (Luis planchó sus camisas de algodón):

[\{CAUSAR/HACER $\}$ (x, [DEVENIR (y, estado)])]

b. Verbo intransitivo no pronominal con interpretación de propiedad (Las camisas de algodón planchan bien):

[PODER (y, [DEVENIR (y, estado)])]

Dos son las características principales que diferencian estas dos estructuras: en primer lugar, la existencia de dos participantes $(x$ e $y)$ en el caso de la variante transitiva eventiva (15a), frente a un único participante $(y)$ en la variante intransitiva con interpretación de propiedad (15b); en segundo lugar, la presencia de un operador de posibilidad (PODER) en la estructura léxico-semántica de la variante intransitiva (15b), que daría cuenta de la lectura de propiedad de esta variante, entendida la propiedad que se predica del único participante como una capacidad para experimentar el cambio de estado físico expresado por el verbo.

Se trata de un análisis léxico-semántico muy esquemático, pero suficientemente explícito para los dos objetivos restantes de este trabajo: realizar una revisión del tratamiento lexicográfico que la variante intransitiva no pronominal con interpretación de propiedad recibe en los principales diccionarios generales de español y, en caso de que este tratamiento no resulte satisfactorio, ofrecer una propuesta de codificación lexicográfica que trate de subsanar las deficiencias encontradas.

\section{TRATAMIENTO LEXICOGRÁFICO EN LOS DICCIONARIOS GENERALES DE ESPAÑOL}

En nuestra revisión del tratamiento lexicográfico que los verbos objeto de estudio reciben en los principales diccionarios generales de español nos centraremos en el Diccionario de uso del español (DUE) de María Moliner; en el DRAE-2001; en el Diccionario de español actual (DEA), de Seco, Andrés y Ramos (1999) y en el Diccionario Salamanca de Gutiérrez Cuadrado (1996). Tal como señalamos en la nota 1, a pesar de que el Diccionario Salamanca es un repertorio lexicográfico dirigido a la enseñanza del español, optamos por incluirlo entre las 
obras revisadas, ya que, como veremos, presenta algunas particularidades interesantes en relación con el tratamiento de los verbos objeto de estudio.

\subsection{DICCIONARIO DE USO DEL ESPAÑOL (1966-1967 / 1998)}

En la primera edición del $D U E$ (1966-1967) no se registra ningún caso de codificación del uso intransitivo no pronominal con significado de propiedad que aquí nos interesa, al menos en lo que respecta a los verbos que presentamos anteriormente en (4).

En cambio, en la segunda edición del DUE (1998) encontramos una diferencia significativa respecto de la primera edición: dentro de la primera acepción transitiva del verbo cerrar se recoge una subacepción intransitiva cuya definición refleja el significado de propiedad que aquí nos interesa ("poder quedar cerrado un receptáculo"). De igual modo, el ejemplo con que se ilustra esta acepción refleja claramente el uso intransitivo no pronominal con interpretación de propiedad (Esta botella de gaseosa no cierra bien, por eso se va el gas). En (16) presentamos la mencionada acepción del verbo cerrar $^{11}$ :

(16) cerrar: [...] tr. o abs. Estar una cosa separando un $\$ lugar de lo que le rodea: 'Una verja cierra el jardín' $\odot$ Estar una cosa impidiendo `la comunicación de un ${ }^{\searrow}$ recinto con el exterior o el paso por un sitio; el complemento es generalmente la abertura tapada o una de las palabras «acceso, entrada, salida», etc.: 'Una gran piedra cierra la boca de la cueva. Una sólida puerta nos cerraba el paso. Unas compuertas cierran la salida del agua'. También puede ser el recinto: 'Este tapón cierra herméticamente la botella'. Cuando se trata de fluidos, también éstos pueden hacer, en lenguaje coloquial, de complemento directo: 'Cierra el agua cuando yo te diga'. Lo que impide el paso puede ser también una persona, un perro, etc.: 'Un guarda les cerró el paso'. $\odot$ Puede usarse también en sentido figurado: 'Este fracaso me cierra otra posibilidad. Su negativa me cerraría el camino para otras gestiones'. $\odot$ intr. Poder quedar cerrado un receptáculo: 'Esta botella de gaseosa no cierra bien, por eso se va el gas'.

11 Respetamos la tipografía de cada uno de los diccionarios revisados. 
Como podemos comprobar, el símbolo circular que aparece tres veces dentro de la primera acepción del verbo cerrar indica la inclusión de una subacepción dentro de una acepción principal.

Cabe cuestionarse por qué otros verbos que claramente permiten un mismo uso intransitivo no pronominal con interpretación de propiedad, como abrir, antónimo de cerrar, u otros semánticamente no relacionados con cerrar, como lavar, planchar o secar, no reciben el mismo tratamiento. Así pues, el $D U E$ no se muestra coherente a la hora de dar cuenta del uso intransitivo no pronominal con interpretación de propiedad de determinados verbos, ya que sólo lo codifica en el caso de $c e-$ rrar, si bien emplea en su definición un verbo modal como poder, que recoge adecuadamente el significado modal de propiedad del uso intransitivo de cerrar que aquí nos interesa. Tras la definición, se ofrece un ejemplo que ilustra el uso de la variante intransitiva de cerrar. Finalmente, cabe destacar que este uso intransitivo de propiedad no aparece codificado como acepción independiente, sino como subacepción dentro de una acepción transitiva eventiva.

\subsection{DiCCIONARIO DE LA LENGUA ESPAÑOLA (DRAE-2001)}

En su edición más reciente, el DRAE-2001 codifica como acepción independiente el uso intransitivo no pronominal con interpretación de propiedad en el caso de dos verbos: cerrar ${ }^{12}$, como ya sucedía en el DUE-1998, y lavar ${ }^{13}$, tal como presentamos en (17):

(17) a. cerrar. [...] $\|$ 27. intr. Dicho de una cosa: cerrarse o poderse cerrar. Este armario, este reloj, este medallón, esta puerta cierra bien o mal, o no cierra. b. lavar. [...] $\|$ 6. intr. Dicho de un tejido: Prestarse más o menos al lavado. Esta cretona lava bien.

12 La primera vez que la Academia recoge esta variante intransitiva de cerrar como acepción independiente es en la edición manual de 1950 de su diccionario.

13 Esta variante intransitiva de lavar aparece recogida por primera vez como acepción independiente en la edición del DRAE de 1970. 
En el caso del verbo abrir, el DRAE-2001 registra su uso intransitivo con la marca "U. t. c. intr." dentro de la segunda acepción transitiva de este verbo, como se observa en (18). Aunque no se registra el significado de propiedad en la definición, el ejemplo que se ofrece para ilustrar el uso intransitivo no pronominal se corresponde justamente con este significado de propiedad (Esta puerta abre bien o abre mal):

(18) abrir. [...] $\|$ 2. Separar del marco la hoja o las hojas de una puerta o ventana, haciéndolas girar sobre sus goznes, o quitar o separar cualquier otra cosa con que esté cerrada una abertura, para que deje de estarlo. U. t. c. intr. y c. prnl. Esta puerta abre bien o abre mal. Abrirse una puerta.

Junto con la objeción ya mencionada en el caso del $D U E$-1998, esto es, el hecho de que se codifique la variante intransitiva no pronominal con interpretación de propiedad sólo en el caso de unos verbos (cerrar, lavar), y no en el caso de otros (secar, planchar, coser, etc.), cabe señalar también que el $D R A E-2001$ no sigue un modelo uniforme a la hora de registrar el uso intransitivo de estos verbos. En dos casos (cerrar y lavar) se consigna el uso intransitivo de propiedad como acepción independiente (véanse (17a) y (17b)), mientras que en el caso de abrir el uso intransitivo no aparece como acepción independiente, sino como marca de uso dentro de una de las acepciones transitivas (véase (18)).

En tercer lugar, en el DRAE-2001 no se sigue un patrón uniforme en la definición de la acepción intransitiva de propiedad de los verbos cerrar y lavar. En lo que respecta a cerrar, tras especificar el tipo de entidad de la que el verbo se predica ("Dicho de una cosa"), se define la acepción intransitiva de propiedad mediante el verbo en forma pronominal (cerrarse) así como mediante la perífrasis modal poderse cerrar, tal como se observa en (17a). En cambio, la acepción intransitiva de propiedad del verbo lavar se define como 'Prestarse más o menos al lavado', definición que aparece igualmente tras la especificación del tipo de entidad de la que el verbo se predica.

Finalmente, hay que destacar que en los tres casos comentados (cerrar, lavar y abrir) en el DRAE-2001 se ofrece un ejemplo que ilustra adecuadamente el uso intransitivo que se está codificando. 


\subsection{DicCionaRio SALAMANCA DE LA LENGUA ESPAÑOLA (1996)}

En el Diccionario Salamanca nos encontramos, junto con los ya mencionados cerrar y lavar, recogidos también por el DRAE-2001, el verbo ajustar con una acepción intransitiva con interpretación de propiedad, tal como se observa en (19):

(19) a. ajustar. [...] $\| v$. intr. / prnl. 11. Estar $<$ una cosa $>$ dentro, encima o alrededor de [otra cosa] de manera que la primera se adapta perfectamente a la segunda: El tapón no ajusta bien en la botella. La llave no se ajusta a este tipo de tuerca.

b. cerrar. [...] $\| v$ intr. / prnl. 17. Estar $<$ una cosa $>$ de manera que no deja ver o tocar su interior: Este armario no (se) cierra bien. 18. Estar <una cosa $>$ de manera que impide el paso. La puerta (se) cierra muy bien.

c. lavar. [...] $\| v$. intr. 6. Ser < un tejido $>$ de fácil o difícil lavado: Esta blusa lava de maravilla. Esta tela lava muy mal.

En este diccionario destaca como novedad el hecho de que, en los casos de ajustar y cerrar, tras la marca de verbo intransitivo figura también la marca de verbo pronominal, con el fin de indicar que estos verbos ofrecen dos posibles construcciones: una intransitiva, otra pronominal. Esta opcionalidad se representa en los ejemplos situando el clítico se entre paréntesis. En el caso del verbo lavar, en cambio, no se codifica esta doble posibilidad. Cabría preguntarse si, en efecto, la variante pronominal y la variante intransitiva son siempre intercambiables en todos los contextos. Como señalamos en nuestro estudio descriptivo (véase el apartado 2.2), parece que determinados adverbios se combinan más frecuentemente con el verbo en forma pronominal que con el verbo en forma intransitiva no pronominal. Nos referimos a una oración como Las camisas de algodón se planchan fácilmente, totalmente aceptable, frente a ?Las camisas de algodón planchan fácilmente, de aceptabilidad algo dudosa, tal como se mostró mediante los ejemplos de (9).

Igualmente hay que señalar que en el Diccionario Salamanca las restricciones de selección semántica que afectan al sujeto se codifican mediante el contorno, situando entre paréntesis en ángulo el tipo de entidad de la que se predica el verbo ("<una cosa>" en el caso de ajustar y cerrar, "<un tejido $>$ " en el caso de lavar). 
En lo que respecta a los aspectos menos acertados, en primer lugar hay que señalar que otros verbos, como abrir, secar, planchar o coser, tampoco aparecen codificados en su uso intransitivo de propiedad en este diccionario. Otro aspecto cuestionable es el hecho de que las definiciones no sigan un patrón homogéneo. Así, encontramos el empleo del verbo estar en el caso de ajustar (19a) y cerrar (19b), pero el verbo ser en el caso de lavar (19c). Finalmente, las definiciones que se ofrecen en el caso de cerrar resultan en exceso detalladas y no se corresponden exactamente con el tipo de ejemplo mencionado.

\subsection{DiCCIONARIO DEL ESPAÑOL ACTUAL (1999)}

Como se puede observar en (20), el número de verbos que se codifican como intransitivos no pronominales con significado de propiedad aumenta sensiblemente en el $D E A$ respecto de los diccionarios revisados anteriormente. Junto con ajustar, cerrar y lavar aparecen abrir, doblar y teñir. Aún así, verbos como planchar o secar no reciben el mismo tratamiento, a pesar de que su uso es similar:

(20) a. abrir: [...] B. intr. [...] Permitir ser abierto [...] [algo, esp. un espacio, una puerta, un cajón, o algo articulado o plegado]. | Aparicio Año 65: Tomó la manija y tiró de ella, pero la puerta no abrió. Aparicio Año 65: Oyó como la cerradura abría. *Este paraguas abre bien.

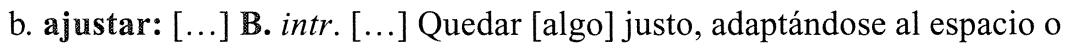
medida que le corresponde. Frec pr. Frec con un compl EN o ci.|Puericultura 14: Se co[n]fecciona [el pañal-braga] con tres telas cogidas con una pretina que ajusta en la cintura.

c. cerrar: [...] B. intr. 21 Pasar a estar incomunicado o separado [algo, esp. un espacio] con respecto al exterior. $T b \mathrm{pr} \mid$ Economía 32: Se mezcla estos ingredientes en un papel blanco y se conservan en una caja o bote que cierre bien. 22 Pasar a estar [un cajón u otro objeto similar] encajado en su hueco. | * Este cajón cierra fatal. 23 Servir [una puerta u otro objeto] para incomunicar o separar un espacio con respecto al exterior.|Cela Alcarria 76: la puerta de la alcoba no cierra, pero eso tampoco importa demasiado. Abc Extra 12.62, 49: Un balcón con unas maderas pintadas de blanco que cerraban muy bien. Aldecoa Gran Sol 37: Patrón, este ojo de buey cierra mal, se filtra el agua. 24 Pasar a estar replegada [una cosa articulada que estaba extendida]. * 
Este paraguas cierra mal. 25 Servir [un objeto, esp. un grifo] para impedir el paso de un fluido. |* Este grifo no cierra.

d. doblar: [...] B. intr. Pasar [algo o alguien] a tener una parte sobre otra o a formar curva o ángulo. Más frec pr. |* El cristal no dobla.

e. lavar: [...] B. intr. [...] Ser lavable [algo, esp. tejido o una prenda]. Frec con los advs BIEN, MAL o equivalentes. | Ya 9.12.72, 3: Los jerseys Crilenka lavan muy fácilmente.

f. teñir: B. intr. Ser [algo] susceptible de ser teñido [1 y 2].| *Este tejido tiñe muy mal.

En el $D E A$ se marcan los subtipos de una categoría gramatical mediante las letras $\mathbf{A}$ y $\mathbf{B}$, en mayúscula y negrita. Esta distinción permite dar cuenta de las alternancias sintácticas que determinan cambios en el significado, como por ejemplo en muchos verbos la capacidad de funcionar como transitivo o como intransitivo. Así, en el apartado B de los verbos objeto de estudio se recoge su uso intransitivo, marcado con la abreviatura intr., a la que siguen diferentes subacepciones. Además, se marca el contorno del verbo y la información sintáctica pertinente en cada caso. A continuación de cada definición figuran bien una o más citas que las documentan, bien un ejemplo de uso. Otro aspecto destacable de este diccionario es que, en alguna ocasión, como en el caso de lavar (20e), se indica que este uso intransitivo con significado de propiedad se da frecuentemente con adverbios como bien o mal.

Entre los aspectos menos acertados se encuentra, por una parte, el hecho de que algunas definiciones sean en exceso detalladas (véase el caso de cerrar, en (20c)) y, por otra, el hecho de que las definiciones no sigan un patrón homogéneo: se emplea en algunas definiciones el verbo de significado modal permitir seguido de una perífrasis pasiva (permitir ser abierto, en (20a)); en otros casos, en la definición se recurre al verbo ser seguido de un adjetivo en -ble, de significado modal (ser lavable, en (20e)); en otras ocasiones, nos encontramos con el verbo ser seguido del adjetivo susceptible con un complemento en el que aparece una perífrasis pasiva (ser susceptible de ser teñido, en (20f)). 


\subsection{CONCLUSIONES DE LA REVISIÓN LEXICOGRÁFICA}

Tras realizar esta revisión del tratamiento que recibe en cuatro diccionarios de español el uso intransitivo de propiedad de verbos como lavar, podemos extraer varias conclusiones.

En primer lugar, los cuatro diccionarios revisados difieren en la nómina de verbos con uso intransitivo no pronominal de propiedad que codifican, aunque cerrar y lavar se encuentran presentes en casi todos. Muchos verbos que también permiten esta variante, como coser, planchar o secar, no aparecen codificados con este uso en ninguno de los diccionarios estudiados.

En segundo lugar, los diccionarios examinados coinciden en presentar ejemplos que ilustran el uso de estos verbos, aunque en el $D E A$ se combinan ejemplos con citas documentadas.

La revisión del tratamiento lexicográfico que reciben los verbos que admiten una variante intransitiva no pronominal con interpretación de propiedad revela, en tercer lugar, que la estructura de la definición no es homogénea ni entre los distintos diccionarios, ni en un mismo diccionario.

Igualmente hemos observado que únicamente el $D E A$ distingue dos macroacepciones, una transitiva (A) y otra intransitiva (B), dentro de la cual se sitúa el uso que aquí estudiamos, como forma de reflejar las alternancias de transitividad con repercusión en el significado de un verbo.

Finalmente, cabe destacar que solamente el $D E A$ incluye en ocasiones una indicación referida al hecho de que la variante intransitiva no pronominal con interpretación de propiedad de estos verbos se combina generalmente con los adverbios bien o mal.

\section{PROPUESTA DE CODIFICACIÓN LEXICOGRÁFICA}

No todos los estudiosos están de acuerdo sobre la cantidad de información gramatical que debe codificarse en un diccionario. En un trabajo ya mencionado sobre el tratamiento lexicográfico de los verbos pronominales, Azorín Fernández y Martínez Linares (2001) se preguntan si un diccionario debe indicar el hecho de que un verbo admite la cons- 
trucción mediopasiva. Estas autoras consideran que la respuesta a la cuestión de cuánta gramática debe contener el diccionario depende del tipo de repertorio lexicográfico que se pretenda elaborar. Por otra parte, lingüistas como Catalá (2000) consideran necesario consignar en el diccionario las alternancias verbales del tipo de la construcción mediopasiva del español.

Por nuestra parte, no nos pronunciaremos sobre la necesidad o no de codificar en un diccionario la posibilidad de que un verbo aparezca en la construcción mediopasiva, ya que no se trata del objeto de estudio de este trabajo. Nos limitaremos a apuntar que, al tratarse de un tipo de construcción sujeta a escasas restricciones en español ${ }^{14}$, serían muchos los verbos que deberían estar marcados como susceptibles de dar lugar a la construcción mediopasiva.

Distinto es, a nuestro entender, el caso que aquí estudiamos: los verbos transitivos del español que permiten una variante intransitiva no pronominal con interpretación de propiedad. Tal como hemos mostrado en el apartado 2.1, sólo admiten esta variante ciertos verbos transitivos con un argumento externo Agente o Causa y un argumento interno afectado físicamente. En el apartado 2.3. afirmamos que esta alternancia entre la variante transitiva eventiva y la variante intransitiva no pronominal con interpretación de propiedad puede ser considerada como un caso de polisemia léxica. Por tanto, para ser coherentes con este análisis, proponemos que la variante intransitiva no pronominal con interpretación de propiedad debe ser codificada en un diccionario, si no como una entrada independiente, sí al menos como una acepción particular de determinados verbos generalmente transitivos y eventivos.

En (21) presentamos nuestra propuesta de codificación lexicográfica de la variante intransitiva no pronominal con interpretación de propiedad de verbos como lavar, planchar o secar. Se trata de un modelo o plantilla que debería ser implementado para cada verbo en concreto:

14 Tratamos brevemente esta cuestión en el apartado 2.2. 
(21) lema: A tr. [...]

B intr. Poder ser + participio [tipo de sujeto]. Frec con los advs BIEN, MAL o equivalentes. | Ejemplo.

Como se observa en (21), adoptamos en nuestro modelo de artículo lexicográfico la convención empleada en el $D E A$ según la cual se distinguen subcategorías dentro de una categoría gramatical empleando las letras mayúsculas $\mathbf{A}$ y $\mathbf{B}$ en negrita. No trataremos aquí la microestructura correspondiente a la acepción transitiva (A). Nos centraremos, en cambio, en la microestructura correspondiente a la acepción intransitiva, marcada como $\mathbf{B}$.

Tras la $\mathbf{B}$ mayúscula, situamos la marca gramatical intr., que nos indica que se trata la variante intransitiva del verbo en cuestión. A continuación, consignamos la definición, que presentaría la misma estructura para todos los verbos de este tipo: poder ser + participio, definición que refleja el significado de propiedad de tipo modal característico de los verbos objeto de estudio. Evitamos el empleo de adjetivos como lavable, utilizados en ocasiones en el $D E A$, porque no se documentan adjetivos en -ble para todos los verbos objeto de estudio. Así, planchable y secable, sin ser formaciones agramaticales, pueden resultar extrañas para el usuario del diccionario por su escasa frecuencia de uso. Por tanto, descartamos el empleo de este tipo de adjetivos derivados, ya que, como hemos mencionado anteriormente, nuestra intención es la de proponer un modelo de definición uniforme para todos los casos concretos de verbos con variante intransitiva no pronominal con interpretación de propiedad.

Mediante el contorno entre corchetes indicamos las restricciones de selección semántica que afectan al sujeto. Tras la definición, se recoge una indicación sobre el uso frecuente de los adverbios bien, mal o equivalentes combinados con esta acepción intransitiva de propiedad. Finalmente, se ofrece un ejemplo que ilustre el uso de esta variante intransitiva con interpretación de propiedad.

En (22) aplicamos este modelo de codificación lexicográfica de la variante intransitiva no pronominal con interpretación de propiedad al caso concreto de planchar: 
(22) planchar: A tr. [...]

B intr. Poder ser planchado [algo, esp una tela]. Frec con los advs BIEN, MAL o equivalentes. $\mid$ El algodón plancha muy bien; en cambio, el lino plancha mal.

Nuestra propuesta de codificación lexicográfica de la variante intransitiva no pronominal con interpretación de propiedad de verbos del tipo de lavar, planchar o secar comparte con el sistema empleado en el $D E A$ la división en subcategorías de las acepciones, la indicación mediante el contorno de las restricciones de selección que afectan al sujeto así como las indicaciones de la frecuente combinación de esta variante intransitiva con adverbios como bien o mal.

Nuestra propuesta difiere del $D E A$, en cambio, en el empleo de un tipo uniforme de definición, consistente en la perífrasis modal pasiva poder ser + participio. De igual modo, nos alejamos del $D E A$ en el hecho de que consideramos que la variante intransitiva con significado de propiedad debe codificarse en el caso de un mayor número de verbos que los tratados en ese diccionario. Al menos todos los verbos recogidos en (4) deberían ser codificados de la manera propuesta, aunque en última instancia serán los criterios de elaboración de cada diccionario - como podría ser el criterio de la frecuencia de aparición de una voz en un determinado corpus, empleado en la elaboración del Diccionario del español usual en México (1996)- los que determinarán qué voces se codifican y en qué acepciones.

\section{CONCLUSIONES}

En este trabajo hemos realizado un estudio descriptivo de la variante intransitiva no pronominal con interpretación de propiedad de algunos verbos del español, en el que hemos llegado a la conclusión de que presentan esta variante ciertos verbos transitivos con un argumento externo Agente o Causa y un argumento interno afectado físicamente. Sin embargo, no todos los verbos que responden a esta caracterización admiten la variante intransitiva no pronominal con interpretación de propiedad aquí estudiada. Así, frente a abrir, cerrar, lavar o planchar, que 
presentan esta posibilidad, se encuentran romper, manchar o arreglar, que carecen de ella.

A continuación hemos llevado a cabo una revisión del tratamiento que esta variante intransitiva no pronominal con significado estativo recibe en los principales diccionarios generales del español ( $D U E$, $D R A E-2001$, Diccionario Salamanca, DEA). Esta revisión lexicográfica ha puesto de manifiesto, por una parte, que los diccionarios difieren en la nómina de verbos codificados; por otra, que la estructura de la definición no es homogénea ni entre los distintos diccionarios, ni en un mismo diccionario; finalmente, que, salvo el $D E A$, no se suele indicar la frecuente aparición de adverbios como bien o mal combinados con la variante intransitiva objeto de estudio.

Tras la revisión lexicográfica de los principales diccionarios generales del español, hemos presentado una propuesta de codificación lexicográfica de la variante intransitiva no pronominal con interpretación de propiedad que presentan algunos verbos de nuestra lengua. Nuestra propuesta se inspira fundamentalmente en el $D E A$, pero se aleja de este diccionario en dos aspectos fundamentales: por una parte, proponemos el empleo de un modelo uniforme de definición; por otra, consideramos que la nómina de verbos codificados con este uso intransitivo con interpretación de propiedad debe ser mayor que la que se registra en el $D E A$.

Queda pendiente de estudio, por una parte, la evolución diacrónica del uso de esta variante intransitiva con significado de propiedad y, por otra, la revisión diacrónica del tratamiento lexicográfico de esta variante. De igual modo, como señalábamos en el apartado 2.1, queda pendiente de estudio la variante intransitiva con interpretación de propiedad de los verbos aquí mencionados como posible rasgo lingüístico asociado a un determinado sociolecto. 


\section{APÉNDICE: DATOS}

A continuación ofrecemos ejemplos del español actual documentados en diccionarios, en el CREA o en otras páginas de Internet, en los que un verbo habitualmente transitivo es empleado en la variante intransitiva no pronominal con interpretación de propiedad:

abrir: Las flores de los gladiolos abren bien cuando se quita el boton que tienen hasta arriba (Luis Lesur, Manual de arreglos florales: una guía paso a paso, México, 1998, apud CREA) ajustar: El tapón no ajusta bien en la botella (apud SALAMANCA). arrugar: [...] si tienes un buen tendedero, no te merece la pena, arruga mucho, tarda mucho en secar y de verdad no compensa (Expertos en decoración responden a tus preguntas. Internet: http://www.todoexpertos.com/categorias/casa_y_jardin/decoracion/Default.aspx?vistaactual=expertos\&pag=).

asar: Las aves de tiro estofadas, preparadas al estilo de Castilla-La Mancha, por ejemplo, asan bien con tintos de cuerpo mediano, con viva acidez (Luis Cañas Crianza 99) que contraste con la salsa del plato ("Es tiempo de caza", La Revista de Todovino, Internet: http://club-abc.todovino.com/revista.jsp?content=revista\&art_id $=33$ )

cerrar: Cuando la tienda, ya montada, no cierra bien, se aflojarán los vientos esquinados opuestos a la entrada (José María de la Poza Lleida, Servicios turísticos, España, 1993, apud CREA).

cocinar: La mayoría de arroces cocinan bien con esta proporción: 1 taza y ? de agua, 1 taza de arroz y 1 cucharita de sal (VV. AA., Cocina cubana, Cuba, 1997, apud CREA).

congelar: Nota: Esta receta no congela bien. Intentamos congelarla y quedó muy líquida la crema de chocolate (Revista Sazón, Internet:http://www.revistasazon.com/archivos/Rev_113/clubleu.htm). cocer: La harina es de color oscuro debido al color de sus cubiertas pero da lugar a un producto que cuece bien y es sabroso y nutritivo (Terralia, Internet: www.terralia.com/revista6/pagina18.htm). descongelar: El frigorífico, de usarlo tan poquísimo, no descongela bien (El Mundo, 31/03/1996: Antonio Burgos, "El fin de los medios", España, apud CREA). 
doblar: El cristal no dobla (apud DEA).

fruncir: Y ahora arreglate bien la pollera, / que me parece que frunce mal ("Alma de organdí", letra de Baltasar de León, Internet: http://www.canciones.pescadores.net/letras/tanguitos/a/De\%20Le on_ALMA\%20DE\%20ORGANDI.htm)

lavar: Esta tela lava muy mal (apud SALAMANCA).

planchar: [...] los dependientes de la tela de ropa masculina, que le hacen la pelota a él y a ti te dicen lo bien que planchan las camisas [...] (Marta Fernández Morales, "Generalizando, que es gerundio", octubre 2003, La Morada-La Revista, Internet: http://bitacora.la-morada.com/Archivos/000227.html).

secar: El frío y la humedad son desfavorables para las colas y los barnices, pues no secan bien y estos últimos adquieren un velo blanquecino difícil de eliminar (María Teresa de la Lastra, Cómo restaurar muebles antiguos, España, 1999, apud CREA).

teñir: Este tejido tiñe muy mal (apud DEA).

\section{BIBLIOGRAFÍA}

Ackema, P. y M. Schoorlemmer (1994): "The middle construction and the syntax-semantics interface", Lingua, 93, pp. 59-90.

—_ (1995): "Middles and non-movement", Linguistic Inquiry, 26/2, pp.173-197.

Azorín Fernández, D. y M. a A. MARTínez LinARes (2000): "El tratamiento lexicográfico de los usos pronominales de los verbos transitivos en los diccionarios monolingües del español actual", en M. Martínez Hernández et al. (eds.), Actas del Congreso Internacional de Semántica. Cien años de investigación semántica: de Michel Bréal a la actualidad (Universidad de La Laguna, 27-31 de octubre de 1997), vol. I, Madrid, Ediciones clásicas, pp. 235-349.

(2001): "Sobre los límites de la gramática en los diccionarios. (Una ojeada a la evolución de las indicaciones gramaticales en los diccionarios monolingües del español)", Estudios de Lingüística de la Universidad de Alicante, 15, pp. 361-379.

Bosque, I. y V. Demonte (dirs.) (1999): Gramática descriptiva de la lengua española, Madrid, Espasa Calpe. 
CANo Aguilar, R. (1981): Estructuras sintácticas transitivas en el español actual, Madrid, Gredos.

CATALÁ TORRES, N. (2000): "De la caracterización eventiva a la definición verbal", en M. Martínez Hernández et al., (eds.), Actas del Congreso Internacional de Semántica. Cien años de investigación semántica: de Michel Bréal a la actualidad (Universidad de La Laguna, 2731 de octubre de 1997), vol. I, Madrid, Ediciones clásicas, pp. 307-318.

Catalá Torres, N,. S. Martí Perelló y S. Vallhonrat Bodas (2002): "Algunas observaciones sobre las construcciones medias en español", Verba, 29, pp. 365-387.

FaGAN, S. M. B. (1988): "The English middle", Linguistic Inquiry, 19/2, pp. 181-203.

- (1992): The syntax and semantics of middle constructions. A study with special reference to German, Cambridge, Cambridge University Press.

FELÍU ARQuiola, E. (2004): "Oraciones medias en español: una aproximación desde la Gramática de las Construcciones", comunicación presentada en el VI Congreso de Lingüistica General, Universidad de Santiago de Compostela, 3-7 de mayo de 2004.

FERnÁNDEZ, A., G. VÁZQUEZ y M. ${ }^{a}$ A. MARTí (2002): “Alternancias diatéticas relacionadas con el aspecto", Verba, 29, pp. 389-402.

GARCíA NeGroni, M. ${ }^{a}$ M. (1996): "La construcción media con se", Filología, 29, pp. 55-81 (reimpresión en C. Sánchez López (ed.), Las construcciones con se, Madrid, Visor, 2002, pp. 275-308) (versión citada).

GutiÉRREZ CuAdrado, J. (dir.) (1996): Diccionario Salamanca de la Lengua Española, Madrid, Santillana-Universidad de Salamanca.

LaRa, L. F. (dir.) (1996): Diccionario del español usual en México, México, El Colegio de México.

Levin, B. (1993): English Verb Classes and Alternations, Chicago, The University of Chicago Press.

MARTíN ZORRAQUINO, M. ${ }^{a}$ A. (1979): Las construcciones pronominales en español: paradigmas y desviaciones, Madrid, Gredos.

Martínez Hernández, M. et al. (eds.) (2000): Actas del Congreso Internacional de Semántica. Cien años de investigación semántica: de Michel Bréal a la actualidad (Universidad de La Laguna, 27-31 de octubre de 1997), vol. I, Madrid, Ediciones clásicas. 
MendikoetXeA, A. (1999): “Construcciones con se: medias, pasivas e impersonales", en I. Bosque y V. Demonte (dirs.), Gramática descriptiva de la lengua española, Madrid, Espasa Calpe, vol. II, pp. 16311722.

Moliner, M. (1992): Diccionario de uso del español, Madrid, Gredos (reimpresión).

(1996): Diccionario de uso del español (versión 1.1, CD-Rom), Madrid, Gredos.

- (19982): Diccionario de uso del español, Madrid, Gredos.

ReAl ACADEMIa Española (200122): Diccionario de la lengua española, Madrid, Espasa-Calpe.

- Corpus de referencia del español actual (CREA) (http://www.rae.es).

RodríGuEZ EsPiñEIRA, M. a J. (1990): “Clases de 'Aktionsart' y predicados habituales en español”, Verba, 17, pp. 171-210.

SÁNCHEZ LÓPEZ, C. (ed.) (2002): Las construcciones con se, Madrid, Visor.

SÁNCHEZ López, C. (2002): "Las construcciones con se. Estado de la cuestión”, en C. Sánchez López (ed.), Las construcciones con se, Madrid, Visor, pp. 13-163.

Seco, M., O. AndRÉs y G. Ramos (1999): Diccionario del español actual, Madrid, Aguilar. 